\title{
Saturated fatty acids enhance osteoclast survival
}

\author{
So-Ra Oh, ${ }^{1, *}$ Ok-Joo Sul, ${ }^{1}{ }^{*}$ Youn-Young Kim, ${ }^{*}$ Hye-Jin Kim, ${ }^{*}$ Rina Yu, ${ }^{\dagger}$ Jae-Hee Suh, ${ }^{\S}$ \\ Hye-Seon Choi ${ }^{2}{ }^{2 *}$ \\ Department of Biological Sciences (BK21 Program),* the Immunomodulation Research Center and \\ Department of Food Science and Nutrition, ${ }^{\dagger}$ University of Ulsan, Ulsan 680-749, Korea; and Department of \\ Pathology, ${ }^{\S}$ Ulsan University Hospital, Ulsan 682-714, Korea
}

\begin{abstract}
Hyperlipidemia and marrow fat are associated with lowering bone density in vivo, suggesting that lipid contributes to bone loss. Using bone marrow-derived macrophages, we investigated the effect of saturated fatty acids (SFA) on osteoclastogenesis. The level of free fatty acids and adiposity in bone marrow was significantly elevated in obese mice. SFA increased osteoclast (OC) survival by preventing apoptosis. SFA caused the production of MIP-1 $\alpha$ and led to activation of nuclear factor (NF)- $\kappa B$ in the OC. The absence of Toll-like receptor 4 (TLR4) or myeloid differentiation factor 88 (MyD88) abolished the survival effect of SFA on OC. Ir Elevated levels of SFA in bone marrow plasma due to obesity enhance OC survival through signaling via TLR4, MyD88, and NF-кB, and at least partly, by inducing MIP-1 $\alpha$ in mature OC.-Oh, S-R., O-J. Sul, Y-Y. Kim, H-J. Kim, R. Yu, J-H. Suh, and H-S. Choi. Saturated fatty acids enhance osteoclast survival. J. Lipid. Res. 2010. 51: 892-899.
\end{abstract}

Supplementary key words osteoclast survival $\bullet$ saturated fatty acid $\bullet$ MIP- $1 \alpha \bullet$ Toll-like receptor $4 \bullet$ myeloid differentiation factor 88

Obesity is considered to be a state of chronic inflammation that leads to metabolic derangements. Actually the relationship between obesity and bone loss is highly controversial. Obesity could be a protective factor due to endogenous estrogen production, but recent findings emphasize the positive relationship between obesity and bone loss. Other factors released from increased adipose tissue mass, free fatty acid (FFA), adipokine, and inflammatory cytokines are also necessary for consideration to evaluate the contribution of obesity to bone metabolism. FFA could be a potent candidate to cause bone loss. Hyperlipidemia is shown to decrease bone formation (1) and increase bone resorption (2). Statin, a lipid-lowering drug, has been demonstrated to increase bone mineral density

This work was supported by Korea (KRF) Grants KRF-2008-314-C00249 and KRF-2009-0066232; by KOSEF Grant R01200700021 08202007; and by Grant A090080 from the Korea Healthcare Technology RE'D Project, Ministry of Health, Welfare, and Family Affairs, Republic of Korea.

Manuscript received 4 December 2009 and in revised form 7 April 2010.

Published, JLR Papers in Press, April 11, 2009

DOI 10.1194/jlr.M800626-JLR200
(BMD) not only by a meta-analysis of clinical studies (3) but also by an experimental animal model (4). Absence of TLR4 increased BMD as well as decreased body fat (5). Epidemiological studies also suggest that BMD is significantly related to serum lipid profiles (6). Verma et al. have demonstrated that marrow fat in bone biopsy samples is increased in osteoporosis (7), and osteoporosis has been connected to increased marrow fat content and decreased un-saturation of fat by studies using in vivo proton magnetic resonance spectroscopy (8).

Increased adipose tissue mass is associated with changes in the endocrine and metabolic function of adipose tissue. FFA is a major secretory product from adipocytes as a result of lipolysis of stored triglycerides. FFA level is elevated in obesity along with various adipocyte factors, which are responsible for low-grade inflammation associated with obesity-related metabolic syndrome $(9,10)$. Saturated fatty acid (SFA) induces the expression of pro-inflammatory genes in macrophages (11), and SFA plays an essential role as signaling molecules in the chronic inflammatory disease associated with obesity (12), although the mechanism by which they influence the development of chronic diseases is not clearly understood.

Osteoclasts (OC), multinucleated giant cells that can resorb bone cells, differentiate from hematopoietic stem cells. OC precursors have properties in common with precursors of the monocyte and macrophage cell lineages, which could cross-talk with adipocytes to reinforce inflammatory conditions (13). They have an important role in physiological bone remodeling and participate in the

\footnotetext{
Abbreviations: BMD, bone mineral density; BMM, bone marrowderived macrophage; D, day; EPA, 17-eicosapentaenoic acid; FFA, free fatty acid; KO, knockout; LPS, lipopolysaccharide; M-CSF, macrophagecolony stimulating factor; MNC, multinucleated cell; MyD88, myeloid differentiation factor 88; NF, nuclear factor; OC, osteoclast; RANKL, receptor activator of nuclear factor- $\mathrm{B}$ ligand; SFA, saturated fatty acid; TLR4, Toll-like receptor 4; TNF, tumor necrosis factor; TRAP, tartrateresistant acid phosphatase.

${ }^{1} \mathrm{~S}-\mathrm{R}$. Oh and O-J. Sul contributed equally to this work.

${ }^{2}$ To whom correspondence should be addressed.

e-mail: hschoi@mail.ulsan.ac.kr
} 
bone destruction associated with chronic inflammatory disease (14).

In the present study we investigated whether obesityinduced FFA in bone marrow environment affects bone metabolism by acting on OC. We found that SFA, such as lauric acid (LA) or palmitic acid (PA), increased OC survival. Using Toll-like receptor 4 (TLR4)-knockout (KO) mice and myeloid differentiation factor 88 (MyD88)-KO mice, we explored the role of TLR4 and MyD88 in the OC survival induced by SFA.

\section{MATERIALS AND METHODS}

\section{Reagents}

Recombinant mouse M-CSF, RANKL, and MIP-1 $\alpha$ were obtained from R and D Systems, Inc. (Minneapolis, MN). Bovine serum albumin (BSA) (fatty acid-free and low endotoxin) was purchased from Sigma Chemical Co. (St. Louis, MN), and the sodium salts of lauric acid, palmitic acid, stearic acid, oleic acid, linoleic acid, and cis-5, 8, 11, 14, 17-eicosapentaenoic acid (EPA) were from Nu-Chek (Eslyan, MN). BSA and all fatty acids within used concentration ranges contained undetectable amounts $(<0.03 \mathrm{EU} / \mathrm{ml})$ of endotoxin by E-TOXATE kit (Sigma Chemical Co.). No significant increase of MIP-1 $\alpha$ was found at $0.03 \mathrm{EU} / \mathrm{ml}$ of endotoxin by mature OC. All free fatty acids (FFA) were solubilized in ethanol as described (11). They were mixed with fatty acid-free and low endotoxin BSA at a molar ratio of $10: 1$ in $0.25 \%$ fetal bovine serum. Fatty acid-albumin complex solution was freshly prepared prior to each experiment. The same volume of vehicle was added to the controls as was added with FFA.

\section{Animals}

Male C57BL/6J mice were rendered obese by ingesting a highfat diet (obese) (45\% calories from lard and soybean oil) (Research Diets Inc.) for 13 weeks starting at 9 weeks of age. Control mice (lean) were fed a control diet (10\% calories from soybean oil). All mice were housed in the specific pathogen-free animal facility of the University of Ulsan, Immunomodulation Research Center (IRC) and were handled in accordance with the Institutional Animal Care and Use Committee of the IRC. Standards were approved by that Committee. Serum levels of FFA were 0.664 and $1.309 \mathrm{mEq} / 1$ for lean $(\mathrm{n}=12)$ and obese $(\mathrm{n}=19)$ mice, respectively. To measure FFA in bone marrow, femora were removed and dissected free of adherent soft tissue. The bone ends were cut, and the marrow cavity was flushed out with $\alpha$-MEM from one end of the bone, using a sterile 21-gauge needle. The FFA content of bone marrow was determined by colorimetric analysis of nonesterified fatty acids using a NEFA kit (Wako Chemicals, Japan). The average bone marrow volume of mice was determined to be $\sim 57 \mu \mathrm{l} /$ femur.

\section{Osteoclast formation}

Bone marrow cells (typically three per preparation) were isolated from 4-5-week-old C57BL/6J mice as described (15). TLR$4^{+/+}(\mathrm{C} 3 \mathrm{H} / \mathrm{HeN})$ and TLR-4 ${ }^{-/-}(\mathrm{TLR} 4-\mathrm{KO})(\mathrm{C} 3 \mathrm{H} / \mathrm{HeJ})$ mice were provided by the Jackson Laboratory. MyD88 ${ }^{-1-}$ (MyD88$\mathrm{KO})(\mathrm{C} 57 \mathrm{BL} / 6$ background) was originally a generous gift from Dr. Shizuo Akira, Osaka University, Japan, and provided by the IRC. The genotypes of offspring were determined by Southern blot analysis of DNA from tail biopsies. Femora and tibiae were aseptically removed and dissected free of adherent soft tissue. The bone ends were cut, and the marrow cavity was flushed out with $\alpha$-MEM from one end of the bone using a sterile 21-gauge needle. The resulting bone marrow suspension was washed twice and added to plates along with M-CSF (20 ng/ml) for $16 \mathrm{~h}$. Nonadherent cells were then harvested and layered on a FicollHypaque gradient. The cells at the interface were collected, washed, resuspended in $\alpha$-MEM containing $10 \%$ FBS, and cultured for two more days with M-CSF, at which time large populations of adherent monocyte/macrophage-like cells had formed on the bottom of the culture plates. The small numbers of nonadherent cells and adherent stromal cells were removed by washing the dishes with PBS and by subsequent incubation for $5 \mathrm{~min}$ in $0.25 \%$ trypsin $/ 0.05 \%$ EDTA, respectively. The adherent bone marrowderived macrophage (BMM) was harvested, seeded at a density of $5 \times 10^{4}$ cells/well in 48-well plates, medium containing M-CSF and RANKL ( $40 \mathrm{ng} / \mathrm{ml}$ ) was added. The medium was replaced on day 2 or 3 . After incubation for the indicated times, the cells were fixed in $10 \%$ formalin for $10 \mathrm{~min}$, and stained for tartrateresistant acid phosphatase (TRAP) as described (15). Numbers of TRAP-positive multinucleated cells (MNC) (three or more nuclei) were scored.

\section{Real time quantitative RT-PCR}

Total RNA was reverse-transcribed with oligo-dT and Superscript I enzyme (Invitrogen, Carlsbad, CA), according to the manufacturer's instructions. Quantitative RT-PCR was carried out using SYBR Green 1 Taq polymerase (Qiagen, Hilden, Germany) and appropriate primers on a DNA Engine Opticon Continuous Fluorescence detection System (MJ Research Inc., Waltham, MA) according to the manufacturer's instructions. Specificities of each primer pair were confirmed by melting curve analysis and agarosegel electrophoresis. The housekeeping GAPDH gene was amplified in parallel with the gene of interest. Relative copy numbers compared with GAPDH was calculated using $2^{-\Delta \Delta \mathrm{Ct}}(16)$. The primer sequences used are as follows: $5^{\prime}$-accatgacactctgcaacca- $3^{\prime}$ and $5^{\prime}$-cccaggtctctttggagtca- $3^{\prime}$ (MIP- $\left.1 \alpha\right) ; 5^{\prime}$-acccagaagactgtggatgg- $3^{\prime}$ and $5^{\prime}$-cacattgggggtaggaacac- $3^{\prime}$ (GAPDH).

\section{ELISA}

To each of the wells containing mature OC was added additional medium containing effectors as indicated. The culture medium was harvested at the indicated times, and the concentration of MIP-1 $\alpha$ determined by a sandwich ELISA using coating antiMIP- $1 \alpha \mathrm{Ab}$ and biotinylated anti-MIP- $1 \alpha \mathrm{Ab}$, as recommended by the supplier ( $\mathrm{R}$ and $\mathrm{D})$.

\section{Apoptosis assay}

OC apoptosis was detected with annexin V staining. OC derived from BMM stimulated with RANKL for $4 \mathrm{~d}$ was washed and restimulated with vehicle, M-CSF and RANKL, lauric acid, or palmitic acid for $18 \mathrm{~h}$. For annexin V staining, cells were harvested and transferred to FACS tubes. After washing, cells were incubated with annexin V FITC (BD Biosciences) in binding buffer (10 mM HEPES/NaOH, pH 7.4, $140 \mathrm{mM} \mathrm{NaCl,} 2.5 \mathrm{mM}$ $\mathrm{CaCl}_{2}$ ) for $15 \mathrm{~min}$ and immediately analyzed with FACSCalibur.

\section{Electrophoretic mobility shift assay (EMSA)}

OC generated by RANKL stimulation of BMM for $4 \mathrm{~d}$ was washed, restimulated with vehicle, M-CSF and RANKL, lauric acid, or palmitic acid for $1 \mathrm{~h}$, and nuclear extracts were prepared. NF-кB-binding studies were performed using double-stranded oligonucleotides (Santa Cruz Biotechnology, Santa Cruz, CA) containing an NF- $\mathrm{B}$ consensus binding site. The oligonucleotide or a variant was end-labeled with $\left[\gamma^{-32} \mathrm{P}\right]$ ATP using T4 polynucleotide kinase (Promega, Madison, WI). Five $\mu \mathrm{g}$ of each nuclear extract was incubated at $30^{\circ} \mathrm{C}$ for $20 \mathrm{~min}$ with $1 \mathrm{ng}$ of 
${ }^{32}$ P-labeled NF-кB probe in $10 \mu \mathrm{l}$ of binding buffer containing 1 $\mu \mathrm{g}$ of poly $(\mathrm{dI} \cdot \mathrm{dC}), 15 \mathrm{mM}$ HEPES, $\mathrm{pH} 7.6,80 \mathrm{mM} \mathrm{NaCl}, 1 \mathrm{mM}$ EGTA, $1 \mathrm{mM}$ dithiothreitol, and $10 \%$ glycerol. DNA-protein complexes were visualized by electrophoresis on a native $5 \%$ polyacrylamide gel, vacuum-drying, and autoradiography using an intensifying screen at $-80^{\circ} \mathrm{C}$.

\section{Statistical analysis}

All values are expressed as means \pm SEM. Student's $t$-test was used to evaluate differences between samples of interest and the corresponding controls. A $P$ value of less than 0.05 was considered statistically significant.

\section{RESULTS}

\section{Osteoclastogenesis is stimulated by SFA}

To investigate whether obesity affects the bone marrow microenvironment, we determined the levels of FFA in the bone marrow of lean and obese mice. As shown in Fig. 1A, the level of FFA in the bone marrow of obese mice was significantly higher than in that of lean mice, suggesting that increased FFA could affect bone metabolism. Consistent with biochemical analyses, histology has demonstrated an increase in bone adiposity in obese mice (Fig. 1B). Adipocyte number was elevated in proximal tibia of obese mice compared with lean mice. Since bone loss is more associated with content of saturated fat than with unsaturated fat (9), we focused on whether SFA plays a role in osteoclastogenesis. We determined the effect of SFA on cultures of BMM free of stromal cells and lymphocytes to investigate the direct action on OC. SFA was added to separate cultures during the indicated period after BMM was incubated with M-CSF and RANKL. Lauric acid (C12:0) significantly increased the number of TRAP-positive MNC formed by the BMM when added on 2-4 day (D) or 3-4 D. Palmitic acid (C16:0) was less potent (Fig. 2A). However, exposure to SFA at early stage did not affect OC formation significantly, suggesting that SFA affects mature OC, rather than early OC precursor.
Since the stimulatory effect of SFA was prominent in the later stage of osteoclastogenesis, we tested whether it affected OC survival. As M-CSF or RANKL is also an OC survival factor (17), we removed cytokines from cultures of differentiated OC by washing them on day 4. The cells were then incubated in the presence or absence of SFA for an additional $18 \mathrm{~h}$. As shown in Fig. 2B, the number of mature OC was reduced dramatically in the absence of cytokines, while the reduction was restored in the presence of M-CSF and RANKL. Treatment with lauric acid or palmitic acid alone prevented some loss of mature OC with maximum effect at $50 \mu \mathrm{M}$, although its effect was less than that of M-CSF and RANKL.

Next, we determined by annexin $\mathrm{V}$ staining whether the effect of SFA on survival was due to an anti-apoptotic action (Fig. 2C). As a positive control we observed fewer annexin $\mathrm{V}$-positive OC in the presence of M-CSF and RANKL than when cytokines were removed. Cells incubated with lauric acid or palmitic acid alone also contained fewer annexin $\mathrm{V}$-positive cells, indicating that SFA protects mature OC from apoptosis.

\section{Enhanced OC survival is mediated by a signal through TLR4 and MyD88}

As SFA-induced COX-2 expression in murine macrophage is mediated by a signaling pathway derived from TLR4 (11) and as OC expresses TLR4 (18), we examined whether SFA stimulates the survival of OC via TLR4. We prepared mature OC from $\mathrm{C} 3 \mathrm{H} / \mathrm{HeJ}$ mice that are defective of TLR4, and compared the response to SFA with that of $\mathrm{C} 3 \mathrm{H} / \mathrm{HeN}$ (wild-type) mice. Lauric acid or palmitic acid alone significantly increased the survival of OC derived from $\mathrm{C} 3 \mathrm{H} / \mathrm{HeN}$ mice, but not in that from $\mathrm{C} 3 \mathrm{H} /$ HeJ mice (Fig. 3A). This indicates that TLR4-mediated signals are essential for the effect of SFA on OC survival. Since signaling through TLR4 involves subsequent recruitment of the adaptor molecule MyD88 (19), we investigated whether MyD88 was also required for SFA signaling using
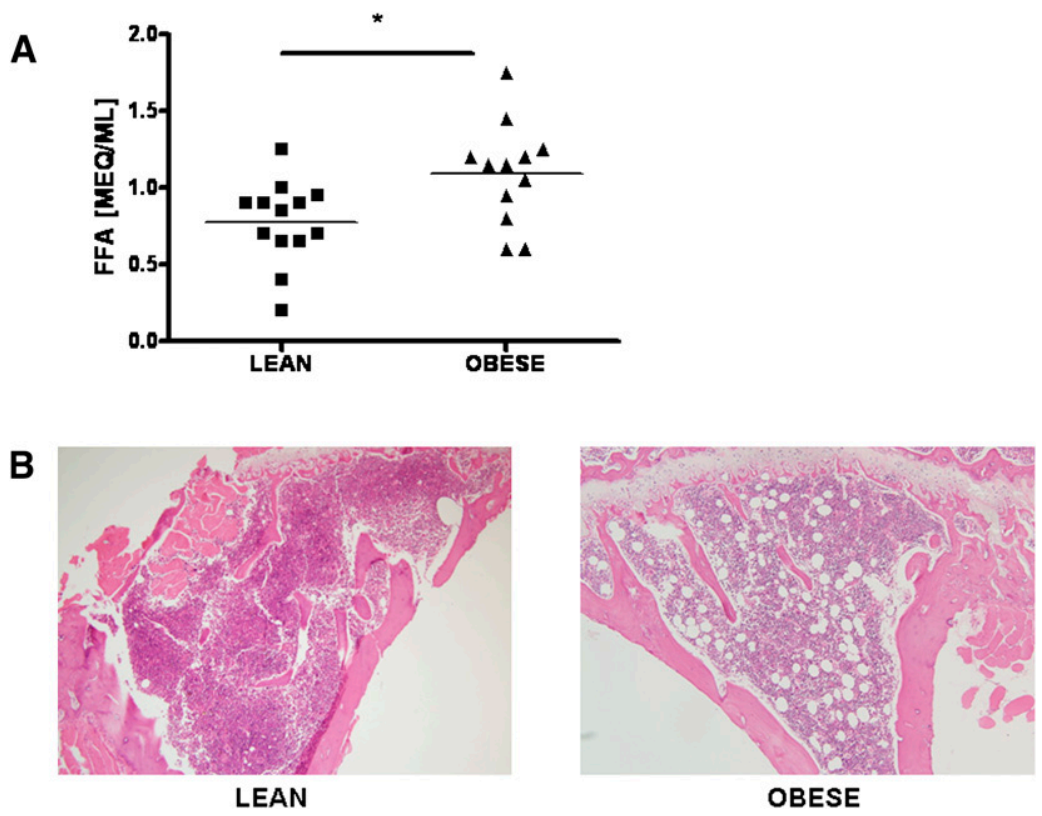

Fig. 1. Increased FFA and adiposity in obese mice. (A) The FFA content of the bone marrow plasma of lean and obese mice was determined by the colorimetric assay for nonesterified fatty acids as described in "Materials and Methods" (*, $P<0.05)$. (B) Representative histology of mouse proximal tibias of lean and obese mice stained with hematoxylin and eosin $(100 \times)$. FFA, free fatty acid. 

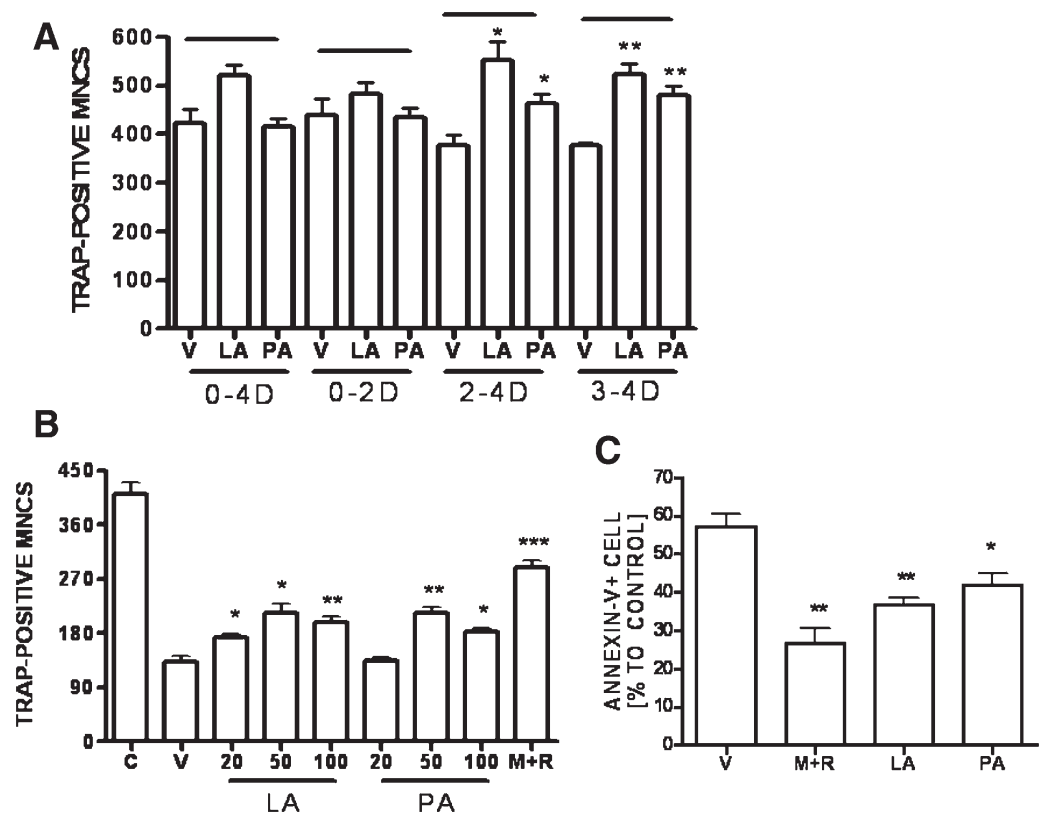

\begin{abstract}
Fig. 2. Effects of SFA on osteoclastogenesis. (A) BMM were prepared as described in "Materials and Methods" and incubated in 48-well plates $\left(5 \times 10^{4}\right.$ cells/well) in the presence of M-CSF $(20 \mathrm{ng} / \mathrm{ml})$ and RANKL $(40 \mathrm{ng} / \mathrm{ml})$ with LA $(50 \mu \mathrm{M})$ or PA $(50 \mu \mathrm{M})$ over the intervals of $0-4 \mathrm{D}, 0-2 \mathrm{D}, 2-4 \mathrm{D}$, and 3-4 D. LA and PA were prepared as described in "Materials and Methods." After $4 \mathrm{~d}$, cells were fixed and stained for TRAP, and the number of TRAP-positive MNC per well was scored. (*, $P<0.05$; **, $P<0.01, \mathrm{n}=3$ ). (B) BMM were stimulated with RANKL for $4 \mathrm{~d}$, extensively washed, and subsequently stimulated with M+R; LA (20, 50, $100 \mu \mathrm{M})$; PA $(20,50,100 \mu \mathrm{M})$; V; or C (day 4 RANKL-stimulated) for $18 \mathrm{~h}(*, P<0.05$; **, $P<0.01$; ***, $P<0.001$, $\mathrm{n}=3$ ). (C) Anti-apoptotic effect of SFA on mature OC. RANKL-induced BMM ( $10^{6}$ cells)-derived mature OC were washed and recultured with V; M+R; LA $(50 \mu \mathrm{M})$; or PA $(50 \mu \mathrm{M})$ for $18 \mathrm{~h}$. Apoptotic cells were stained with annexin V FITC, analyzed with FACSCalibur ( $*, P<0.05$; ** $P<0.01, \mathrm{n}=3$ ). BMM, bone marrowderived macrophage; C, control; D, day; LA, lauric acid; M+R, M-CSF and RANKL; MNC, multinucleated cell; OC, osteoclast; PA, palmitic acid; SFA, saturated fatty acid; TRAP, tartrate-resistant acid phosphatase $\mathrm{V}$, vehicle.
\end{abstract}

MyD88-KO mice. As shown in Fig. 3B, neither lauric acid nor palmitic acid increased OC survival in the absence of MyD88, whereas M-CSF and RANKL could. As signaling by the TLR complex induces activation of NF-кB (20) and because many factors that promote OC survival activate the transcription factor NF-kB (21), we examined the effect of lauric acid or palmitic acid on NF- $\mathrm{B}$ activation. RANKL restimulation induced NF-кB DNA binding activity in mature OC from C57BL/6 mice (wild-type) that had been thoroughly washed. Lauric acid or palmitic acid also activated NF- $\mathrm{KB}$ (lane 4,5) to a similar extent as RANKL (lane 6) (Fig. 3C). However, NF-кB activation upon SFA stimulation was not found in mature OC from MyD88-KO mice and C3H/HeJ (TLR4-KO) mice (Fig. 3D).

\section{MIP-1 $\alpha$ generated by mature OC in response to SFA is responsible for the increased OC survival}

To investigate the factors enhancing OC survival, we first measured the expression of MIP-1 $\alpha$. The induction of MIP-1 $\alpha$ transcripts in mature OC was significantly upregulated after $3 \mathrm{~h}$ of lauric acid addition. Palmitic acid also increased MIP- $1 \alpha$ transcripts, although to a lesser degree (Fig. 4A). To determine the effect of a wide range of FFA using the most sensitive assay, we determined the expression of the MIP- $1 \alpha$ tran- script (Fig. 4B). The effect of stearic acid (C18:0) was more intense than that of palmitic acid (C16:0). Oleic acid (C18:1), linoleic acid (C18:2), and EPA had no significant effect.

Next we examined the level of MIP-1 $\alpha$ secreted by mature OC. As shown in Fig. 4C, mature OC produced a significantly higher level of MIP-1 $\alpha$ after addition of lauric acid or palmitic acid than after addition of the vehicle. The OC survival factors, M-CSF and RANKL, also increased expression of MIP-1 $\alpha$, suggesting a role of MIP-1 $\alpha$ in the elevated OC survival.

We also tested whether SFA affects the expression of receptor for MIP-1 $\alpha$. No increased expression of CCR1 or CCR5 in mature OC was observed in response to lauric acid (data not shown). Because SFA did not change the expression level of receptor for MIP-1 $\alpha$ on mature OC, we examined whether the enhanced OC survival due to SFA was the result of increased MIP-1 $\alpha$. Treatment with MIP-1 $\alpha$ alone prevented the loss of mature OC. Addition of neutralizing $\mathrm{Ab}$ against MIP- $1 \alpha$ to cultures of lauric acid- or palmitic acid-treated OC prevented the increase in OC survival, whereas control IgG had no significant effect (Fig. 4D). However, blockade of MIP-1 $\alpha$ in lauric acid- or palmitic acid-treated OC showed modest, but significantly enhanced OC survival, compared to vehicle-treated OC. Taken 
A
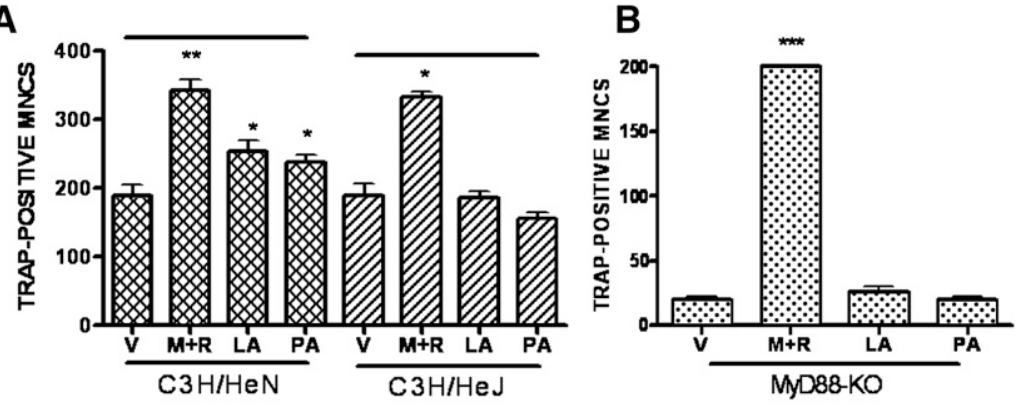

C

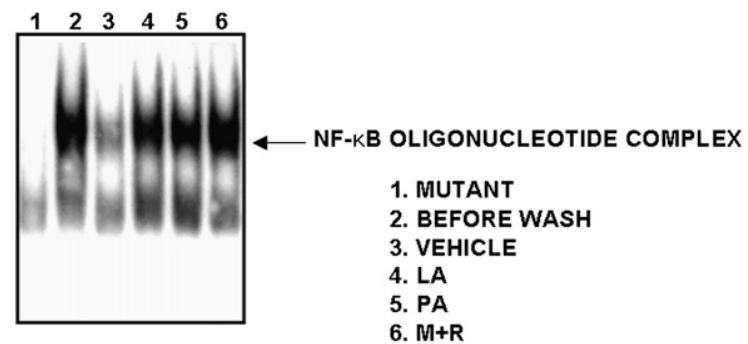

D
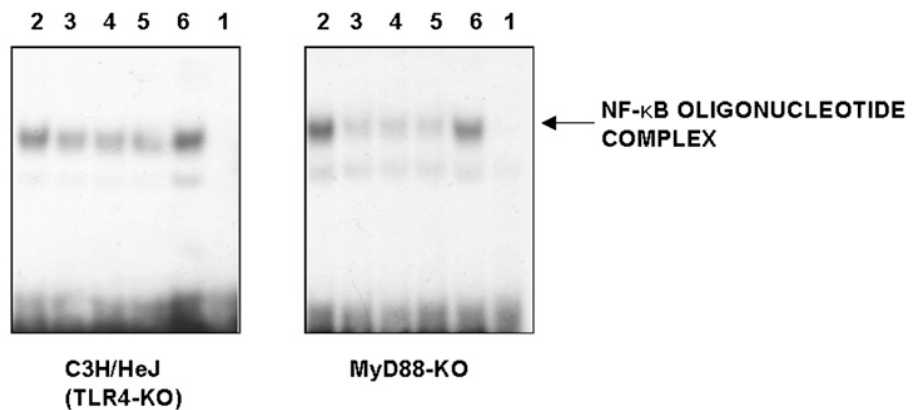

Fig. 3. SFA affects OC survival via TLR4 and MyD88, and induces NF- $\mathrm{B}$ activation. BMM $\left(5 \times 10^{4}\right.$ cells $)$ from $\mathrm{C} 3 \mathrm{H} / \mathrm{HeN}, \mathrm{C} 3 \mathrm{H} / \mathrm{HeJ}$ (A), and MyD88-KO (B) were stimulated with M-CSF $(20 \mathrm{ng} / \mathrm{ml})$ and RANKL (40 ng/ml) for $4 \mathrm{~d}$, extensively washed, and subsequently stimulated with M+R; LA $(50 \mu \mathrm{M})$; PA (50 $\mu \mathrm{M})$; or V for $18 \mathrm{~h}(*, P<0.05$; **, $P<0.01, \mathrm{n}=3)$. OC was generated by M-CSF and RANKL stimulation of BMM $\left(5 \times 10^{6}\right.$ cells $)$ from C57BL/6 (C), C3H/HeJ or MyD88-KO (D) for $4 \mathrm{~d}$, washed, and restimulated with V (lane 3); LA (50 $\mu \mathrm{M}$, lane 4); PA (50 $\mu \mathrm{M}$, lane 5 ); or RANKL (lane 6) for $1 \mathrm{~h}$. RANKL-stimulated OC on day 4 (lane 2) and a variant NF-кB oligomer (lane 1) were used as positive and negative controls, respectively. Similar results were obtained in three independent experiments. LA, lauric acid; $\mathrm{M}+\mathrm{R}$, M-CSF and RANKL; M-CSF, macrophage-colony stimulating factor; MyD88, myeloid differentiation factor 88; OC, osteoclast; PA, palmitic acid; RANKL, receptor activator of nuclear factor-кB ligand; SFA, saturated fatty acid; TLR4, Toll-like receptor 4; V, vehicle. together, these findings indicate that MIP- $1 \alpha$ is a positive regulator of OC survival and that the stimulation of OC survival by SFA is due to an increased level of MIP-1 $\alpha$.

\section{DISCUSSION}

We have demonstrated that the level of FFA in bone marrow is significantly elevated along with marrow adiposity in obese mice, although the degree of increase in bone marrow was slightly lower than that in serum. Although there is no direct evidence that FFA induces bone loss, FFA can be a potential candidate for bone loss. The metabolic syndromes associated with increased level of FFA, diabetes, and atherosclerosis have been demonstrated to be highly related to bone loss $(9,13)$. More alveolar bone loss is associated with the Goto-Kakizaki rat, a type 2 diabetes rat (22), and the Zucker fatty rat, a known model of prediabetes (23). Lower BMD and rapid bone loss is associated with the advanced aortic calcification, a surrogate marker for atherosclerosis, in postmenopausal women (24), and a graded association is also found between atherosclerotic vascular calcification and vertebral bone loss (25). FFA also causes inflammation by activation of JNK and IKK $\beta$ in muscle and liver (9) as well as macrophages (12). Macrophages not only play a critical role as inflammatory mediators in metabolic syndromes (13), but they also participate in the bone loss associated with chronic inflammatory disease (14). Indeed we have shown that SFA stimulates osteoclastogenesis by acting on mature OC, suggesting it could play a critical role in inducing bone loss along with obesity.

Contrary to our results, inhibition or no significant effect of OC formation by SFA in murine macrophage cell line, RAW264.7 cells, has been reported $(26,27)$. However, they did not distinguish the effect of SFA at specific stage. We have presented evidence that SFA is most effective in increasing osteoclastogenesis at a late stage of $\mathrm{OC}$ formation; it enhances OC survival by inhibiting apoptosis of the mature OC via a signal though TLR4, MyD88, and NF-кB. To see whether SFA stimulated the survival of OC through TLR4, mature OC were prepared from TLR4-KO mice. SFA increased the survival of OC derived from wildtype mice of the same strain but not from the TLR4-KO mice, indicating that TLR4-mediated signals are essentially involved in the OC survival promoted by SFA. TLR recognizes not only conserved pathogen-associated molecular patterns of invading microorganisms but also nonmicrobial molecules (28). SFA-induced COX-2 in macrophages has been inhibited by a dominant-negative mutant of TLR4 (11). Removal of SFA or its replacement with unsaturated fatty acids on LPS, a TLR agonist, abolishes its activities and turns it into an antagonist (29), suggesting that SFA is responsible for the effects of TLR agonists. The 

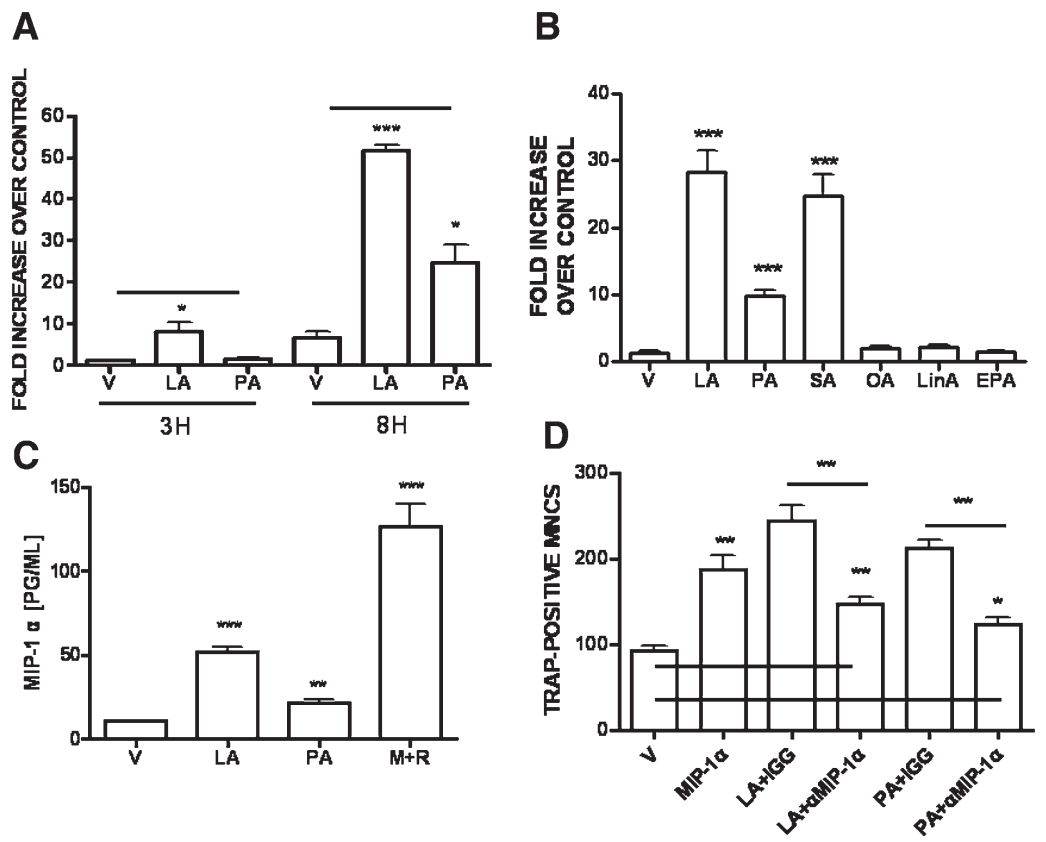

\begin{abstract}
Fig. 4. Induction of MIP- $1 \alpha$ by OC. BMM $\left(2 \times 10^{6}\right.$ cells $)$ was stimulated with RANKL $(40 \mathrm{ng} / \mathrm{ml})$ and M-CSF $(20 \mathrm{ng} / \mathrm{ml})$ for $4 \mathrm{~d}$, extensively washed, and subsequently stimulated with $\mathrm{V}$; LA $(50 \mu \mathrm{M})$; PA $(50 \mu \mathrm{M})$; or $\mathrm{M}+\mathrm{R}$ for $3 \mathrm{~h}, 8 \mathrm{~h}(\mathrm{~A})$, or $18 \mathrm{~h}(\mathrm{C})$. BMM was treated as above, subsequently stimulated with $\mathrm{V}$ or $50 \mu \mathrm{M}$ of LA, PA, SA, OA, LinA, or EPA for $8 \mathrm{~h}$ (B). Total RNA was extracted and subjected to semi-quantitative PCR analysis $(\mathrm{A}, \mathrm{B}) .(*, P<0.05$; ***, $P<0.001, \mathrm{n}=3$ ) The levels of MIP- $1 \alpha$ in the supernatants were measured by ELISA $(\mathrm{C})$. $(* *, P<0.01 ; * * *, P<0.001, \mathrm{n}=3)$. (D) BMM $\left(5 \times 10^{4}\right.$ cells $)$ was stimulated with RANKL and M-CSF for $4 \mathrm{~d}$, extensively washed, and subsequently stimulated with V; MIP-1 $\alpha$ (20 ng/ml); LA (50 $\mu$ M); or PA $(50 \mu \mathrm{M})$. Neutralizing anti-MIP- $1 \alpha \mathrm{Ab}(0.2 \mu \mathrm{g} / \mathrm{ml})$ or control IgG was added simultaneously with LA- or PA-treated BMM to determine the role of MIP- $1 \alpha$ in LA- or PA-stimulated OC survival for $18 \mathrm{~h}(*, P<0.05$; $* *, P<0.01, \mathrm{n}=3)$. Neutralization of MIP-1 $\alpha$ in LA-treated (**, $P<0.01, \mathrm{n}=3$ ) or PA-treated culture $(*, P<$ $0.05, \mathrm{n}=3$ ) was significantly different from vehicle-treated one. BMM, bone marrow-derived macrophage; EPA, 17-eicosapentaenoic acid; LA, lauric acid; LinA, linoleic acid; M+R, M-CSF and RANKL; M-CSF, macrophage-colony stimulating factor; OA, oleic acid; PA, palmitic acid; RANKL, receptor activator of nuclear factor-кB ligand; SA, stearic acid; V, vehicle.
\end{abstract}

role of TLR in osteoclastogenesis is controversial, but the difference could be due to the stage that OC was exposed to TLR agonists. TLR stimulation inhibits OC formation (30), whereas it enhances OC survival (18). Actually in vivo excessive bone resorption by $\mathrm{OC}$ in inflammatory bone diseases is related to bacterial infection, probably as a consequence of activation of TLR (31).

Conserved cytoplasmic regions of TLR associate with MyD88, which is also an adaptor molecule for IL-1 and LPS (19). We have also shown that SFA alone did not promote the survival of OC from MyD88-KO mice, suggesting that MyD88 is required for signaling from SFA. In addition, the number of OC was somewhat lower in vehicletreated cultures from MyD88-KO mice (Fig. 3B) than in that from wild-type mice (Fig. 2B), indicating that OC survival could be enhanced by endogenous molecules signaling through MyD88. However, the absence of TLR4 did not change basal level of OC significantly (Fig. 3A). It could be explained by the redundancy of TLR. TLR forms a complex with MyD88, IRAK, and TRAF6 (28). Signaling by the latter induces activation of NF-KB (20), and increased OC survival is associated with activation of NF-кB (20). Our data also demonstrate that SFA causes activation

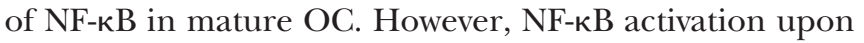

SFA stimulation was absent in TLR4-deficient cells as well as MyD88-deficient ones, indicating that NF- $\mathrm{B}$ activation by SFA is due to a signal through TLR4 and MyD88. We cannot exclude the possibility of a delayed response of SFA, as we do not perform kinetics of NF-кB activation. NF- $\mathrm{KB}$ activation by LPS is delayed, but not absent, in MyD88-deficient cells, suggesting that certain LPS-induced responses do not require MyD88 (32).

We showed that exogenously added SFA affects osteoclastogenesis induced by RANKL by modulating the level of MIP- $1 \alpha$, although it was not demonstrated clearly whether MIP- $1 \alpha$ is a downstream molecule of the above signaling pathway. Our data indicate that MIP- $1 \alpha$ is a positive regulator of $\mathrm{OC}$ survival and that the enhanced $\mathrm{OC}$ survival of SFA-treated OC may be at least partly due to the increased level of MIP- $1 \alpha$. We also determined the effect of a variety of FFA on inducing MIP- $1 \alpha$. SFA had an effect, but it is hard to tell the relationship between the magnitude of effect and chain length because of the small sample size. On the contrary, no significant effect was found with unsaturated fatty acids, suggesting that the response is due to SFA. Although SFA had modest effect on OC survival, it was efficient in induction of MIP- $1 \alpha$ in a comparable degree. This apparent discrepancy in the efficacy 
of SFA according to OC survival and its effect on MIP-1 $\alpha$ induction could be explained by the possibilities that SFA modulates OC survival through production of negative regulators as well as positive ones, including MIP- $1 \alpha$. Our present results showed that MIP-1 $\alpha$ blockade in lauric acid- or palmitic acid-treated OC did not restore the response of baseline, also suggesting a contribution of other factors induced by SFA. Recent studies suggest that chemokines play roles in osteoclastogenesis in vivo and in vitro. In vivo mouse model of inhibition of human myeloma bone disease by MIP- $1 \alpha$, an anti-sense construct markedly reduced MIP- $1 \alpha$ in marrow plasma and decreased bone destruction (33). Moreover addition of a neutralizing $\mathrm{Ab}$ to MIP-1 $\alpha$ to human bone marrow cultures blocked the enhancement of OC formation induced by marrow plasma from patients with active multiple myeloma (34). Our previous data showing increased osteoclastogenesis in $\mathrm{BALB} / \mathrm{c}$ mice, compared to that in C57BL/ 6 mice also suggested that MIP-1 $\alpha$ is a potent osteoclastogenic factor for BMM. MIP-1 $\alpha$ affected not only early precursors but also mature OC by preventing apoptosis (35), and a similar effect has been observed with other chemokines. SDF-1 prevented mature OC from undergoing apoptosis by altering the ratio of expression of Bcl-2 family members (36). MIP-1 $\gamma$, which is upregulated in OC derived from RANKL-stimulated BMM, also promoted the survival of mature OC by activating NF- $\mathrm{B}$ (21). In our previous studies, neutralization of MIP-1 $\alpha$ suppressed OC survival by M-CSF and RANKL, suggesting a critical role of MIP-1 $\alpha$ in OC survival (35). Our present results also showed that M-CSF and RANKL induce OC survival in the absence of TLR4 and MyD88, suggesting that they did not share common signaling pathways with SFA, even with MIP-1 $\alpha$ as a convergent molecule for OC survival.

\section{CONCLUSION}

We found that SFA promoted OC survival by preventing apoptosis. The stimulation of OC survival in SFA-treated OC was caused by an increased level of MIP- $1 \alpha$ and a signal via TLR4, MyD88, and NF-кB activation. Thus, the signaling pathway from SFA has both physiological and pathophysiological implications. The induction of MIP-1 $\alpha$ by SFA may be an important component of OC survival. MIP- $1 \alpha$ is also expressed in primary cultures of preadipocytes and upregulated during adipocyte differentiation (37), suggesting the occurrence of autocrine and paracrine signaling in OC. In the obese state, OC will tend to be more affected by enhanced FFA and to produce MIP$1 \alpha$ or be exposed to it by adjacent cells. The enhanced level of MIP-1 $\alpha$ may be not only chemotactic for monocytes but also promote OC survival, thus playing a role in the cross talk between bone and fat metabolism. Further studies of the intracellular signaling pathway of SFA should help identify therapeutic targets that may reduce elevated FFA levels associated with obesity-induced metabolic diseases.jW
The authors thank Dr. Shizuo Akira for providing MyD88knockout mice.

\section{REFERENCES}

1. Parhami, F., Y. Tintut, W. G. Beamer, N. Gharavi, W. Goodman, and L. L. Demer. 2001. Atherogenic high-fat diet reduces bone mineralization in mice. J. Bone Miner. Res. 16: 182-188.

2. Tintut, Y., S. Morony, and L. L. Demer. 2004. Hyperlipidemia promotes osteoclastic potential of bone marrow cells ex vivo. Arterioscler. Thromb. Vasc. Biol. 24: e6-e10.

3. Uzzan, B., R. Cohen, P. Nicolas, M. Cucherat, and G. Y. Perret. 2007. Effects of statins on bone mineral density: a meta-analysis of clinical studies. Bone. 40: 1581-1587.

4. Gutierrez, G. E., J. R. Edwards, I. R. Garrett, J. S. Nyman, B. McCluskey, G. Rossini, A. Flores, D. B. Neidre, and G. R. Mundy. 2008. Transdermal lovastatin enhances fracture repair in rats. J. Bone Miner. Res. 23: 1722-1730.

5. Johnson, G. B., B. L. Riggs, and J. L. Platt. 2004. A genetic basis for the "Adonis" phenotype of low adiposity and strong bones. FASEB J. 18: 1282-1284.

6. Cui, L-H., M-H. Shin, E-K. Chung, Y-H. Lee, S-S. Kweon, K-S. Park, and J-S. Choi. 2005. Association between bone mineral densities and serum lipid profiles of pre-and post-menopausal rural women in South Korea. Osteoporos. Int. 16: 1975-1981.

7. Verma, S., J. H. Rajaratnam, J. Denton, J. A. Hoyland, and R. J. Byers. 2002. Adipositic proportion of bone marrow is inversely related to bone formation in osteoporosis. J. Clin. Pathol. 55: 693-698.

8. Yeung, D. K. W., J. F. Griffith, G. E. Antonio, F. K. H. Lee, J. Woo, and P. C. Leung. 2005. Osteoporosis is associated with increased marrow fat content and decreased marrow fat unsaturation: a proton MR spectroscopy study. J. Magn. Reson. Imaging. 22: 279-285.

9. Ferrante, A. W., Jr. 2007. Obesity-induced inflammation: a metabolic dialogue in the language of inflammation. J. Intern. Med. 262: 408-414.

10. Boden, G. 2008. Obesity and free fatty acids. Endocrinol. Metab. Clin. North Am. 37: 635-646.

11. Lee, J. Y., K. H. Sohn, S. H. Rhee, and D. Hwang. 2001. Saturated fatty acids, but not unsaturated fatty acids, induce the expression of cyclooxygenase-2 mediated through Toll-like receptor 4. J. Biol. Chem. 276: 16683-16689.

12. Nguyen, M. T., S. Favelyukis, A-K. Nguyen, D. Reichart, P. A. Scott, A. Jenn, R. Liu-Bryan, C. K. Glass, J. G. Neels, and J. M. Olefsky. 2007. A subpopulation of macrophages infiltrates hypertropic adipose tissue and is activated by free fatty acids via toll-like receptors 2 and 4 and JNK-dependent pathways. J. Biol. Chem. 282: 35279-35292.

13. Wellen, K. E., and G. S. Hotamisligil. 2005. Inflammation, stress, and diabetes. J. Clin. Invest. 115: 1111-1119.

14. Roodman, G. D. 1996. Advances in bone biology: the osteoclast. Endocr. Rev. 17: 308-332.

15. Shin, H. H., J. E. Lee, E. A. Lee, B. S. Kwon, and H. S. Choi. 2006. Enhanced osteoclastogenesis in 4-1BB-deficient mice caused by reduced interleukin-10. J. Bone Miner. Res. 21: 1907-1912.

16. Livak, K. J., and T. D. Schmittgen. 2001. Analysis of relative gene expression data using real-time quantitative PCR and the $2^{-\Delta \Delta \mathrm{Ct}}$ method. Methods. 25: 402-408.

17. Glantschnig, H., J. E. Fisher, G. Wesolowski, G. A. Rodan, and A. A. Reszka. 2003. M-CSF, TNF- $\alpha$ and RANK ligand promote osteoclast survival by signaling through mTOR/S6 kinase. Cell Death Differ. 10: $1165-1177$.

18. Itoh, K., N. Udagawa, K. Kobayashi, X. Li, M. Takami, N. Okahashi, N. Nishihara, and N. Takahashi. 2003. Lipopolysaccharide promotes the survival of osteoclast via toll-like receptor 4, but cytokine production of osteoclasts in response to lipopolysaccharide is different from that of macrophages. J. Immunol. 170: 3688-3695.

19. Adachi, O., T. Kawai, K. Takeda, M. Matsumoto, H. Tsutsui, M. Sakagami, K. Nakanishi, and S. Akira. 1998. Targeted disruption of the MyD88 gene results in loss of IL-1- and IL-18-mediated function. Immunity. 9: 143-150.

20. Wong, B. R., R. Joisen, S. Y. Lee, M. Vologodskaia, R. M. Steinman, and Y. Choi. 1998. The TRAF family of signal transducers mediates NF-кB activation by TRANCE receptor. J. Biol. Chem. 273: 28355-28359. 
21. Okamatsu, Y., D. Kim, R. Battaglino, H. Sasaki, U. Spate, and P. Stashenko. 2004. MIP-1 $\gamma$, promotes receptor activator of NF-кB ligandinduced osteoclast formation and survival. J. Immunol. 173: 2084-2090.

22. Pontes Andersen, C. C., K. Buschard, A. Flyvberg, K. Stoltze, and P. Holmstrup. 2006. Periodontitis deteriorates metabolic control in type 2 diabetic Goto-Kakizaki rats. J. Periodontol. 77: 350-356.

23. Pontes Andersen, C. C., A. Flyvberg, K. Buschard, and P. Holmstrup. 2007. Periodontitis is associated with aggrevation of prediabetes in Zucker fatty rats. J. Periodontol. 78: 559-565.

24. Bagger, Y. Z., L. B. Tanko, P. Alexandersen, G. Qin, and C. Christiansen. 2006. Radiographic measure of aorta calcification is a site-specific predictor of bone loss and fracture risk at the hip. J. Intern. Med. 259: 598-605.

25. Schulz, E., K. Arfai, X. Liu, J. Sayre, and V. Gilsanz. 2004. Aortic calcification and the risk of osteoporosis and fractures. J. Clin. Endocrinol. Metab. 89: 4246-4253.

26. Cornish, J., A. MacGibborn, J. Lin, M. Watson, P. C. Tong, J. E. Dunford, Y. van der Does, G. A. Williams, A. B. Grey, D. Naot, et al. 2008. Modulation of osteoclastogenesis by fatty acids. Endocrinology. 149: 5688-5695.

27. Rahman, M. M., A. Bhattacharya, and G. Fernandes. 2006. Conjugated linoleic acid inhibits osteoclast differentiation of RAW264.7 cells by modulating RANKL signaling. J. Lipid Res. 47: 1739-1748.

28. Ulevitch, R. J. 2000. Molecular mechanisms of innate immunity. Immunol. Res. 21: 49-54.

29. Kitchens, R. L., R. J. Ulevitch, and R. S. Munford. 1992. Lipopolysaccharide (LPS) partial structures inhibit responses to LPS in a human macrophage cell line without inhibiting LPS uptake by a CD14-mediated pathway. J. Exp. Med. 176: 485-494.
30. Takami, M., N. Kim, J. Rho, and Y. Choi. 2002. Stimulation of tolllike receptors inhibits osteoclast differentiation. J. Immunol. 169: 1516-1523.

31. Nair, S. P., S. Meghji, M. Wilson, K. Reddi, P. White, and B. Henderson. 1996. Bacterially induced bone destruction: mechanisms and misconceptions. Infect. Immun. 64: 2371-2380.

32. Kawai, T., O. Adachi, T. Ogawa, K. Takeda, and S. Akira. 1999. Unresponsiveness of MyD88-deficient mice to endotoxin. Immunity. 11: 115-122.

33. Choi, S. J., Y. Oba, Y. Gazitt, M. Alsina, J. Cruz, J. Anderson, and G. D. Roodman. 2001. Antisense inhibition of macrophageinflammatory protein-1 $\alpha$ blocks bone destruction in a model of myeloma bone disease. J. Clin. Invest. 108: 1833-1841.

34. Choi, S. J., J. C. Cruz, F. C. Craig, H. Chung, R. D. Devlin, D. Roodman, and M. Alsina. 2000. Macrophage inflammatory protein$1 \alpha$ is a potential osteoclast stimulatory factor in multiple myeloma. Blood. 96: 671-675.

35. Lee, J. E., H. H. Shin, E. A. Lee, T. V. Phan, and H. S. Choi. 2007. Stimulation of osteoclastogenesis by enhanced level of MIP-1 $\alpha$ in BALB/c mice. Exp. Hematol. 35: 1100-1108.

36. Wright, L. M., W. Maloney, X. Yu, L. Kindle, P. Collins-Osdoby, and P. Osdoby. 2005. Stromal cell-derived factor-1 binding to its chemokine receptor CXCR4 on precursor cells promotes the chemotactic recritment, development and survival of human osteoclsts. Bone. 36: 840-853.

37. Gerhardt, C. C., I. A. Romero, R. Cancello, L. Camoin, and A. D. Strosberg. 2001. Chemokines control fat accumulation and leptin secretion by cultured human adipocytes. Mol. Cell. Endocrinol. 175: 81-92. 